\title{
BMJ Open Cohort profile: Indian Network of Population-Based Surveillance Platforms for Influenza and Other Respiratory Viruses among the Elderly (INSPIRE)
}

Anand Krishnan, ${ }^{1}$ Lalit Dar, ${ }^{2}$ Ritvik Amarchand, ${ }^{1}$ Aslesh Ottapura Prabhakaran, ${ }^{3}$ Rakesh Kumar, ${ }^{1}$ Prabu Rajkumar (1) , ${ }^{4}$ Suman Kanungo, ${ }^{5}$ Sumit Dutt Bhardwaj, ${ }^{6}$ Avinash Choudekar, ${ }^{2}$ Varsha Potdar, ${ }^{6}$ Alok Kumar Chakrabarti, ${ }^{5}$ CP Girish Kumar, ${ }^{4}$ Giridara Gopal Parameswaran (1) , ${ }^{1}$ Shivram Dhakad, ${ }^{2}$ Byomkesh Manna, ${ }^{5}$ Ashish Choudhary, ${ }^{2}$ Kathryn E Lafond, ${ }^{7}$ Eduardo Azziz-Baumgartner, ${ }^{7}$ Siddhartha Saha ${ }^{3}$

To cite: Krishnan A, Dar L, Amarchand $\mathrm{R}$, et al. Cohort profile: Indian Network of Population-Based Surveillance Platforms for Influenza and Other Respiratory Viruses among the Elderly (INSPIRE). BMJ Open 2021;11:e052473. doi:10.1136/ bmjopen-2021-052473

- Prepublication history and additional supplemental material for this paper are available online. To view these files, please visit the journal online (http://dx.doi.org/10.1136/ bmjopen-2021-052473).

Received 19 April 2021 Accepted 13 September 2021

Check for updates

(C) Author(s) (or their employer(s)) 2021. Re-use permitted under CC BY-NC. No commercial re-use. See rights and permissions. Published by BMJ.

For numbered affiliations see end of article.

Correspondence to

Dr Anand Krishnan;

anand.drk@gmail.com

\section{ABSTRACT}

Purpose We describe here a multicentric communitydwelling cohort of older adults ( $\geq 60$ years of age) established to estimate incidence, study risk factors, healthcare utilisation and economic burden associated with influenza and respiratory syncytial virus (RSV) in India. Participants The four sites of this cohort are in northern (Ballabgarh), southern (Chennai), eastern (Kolkata) and western (Pune) parts of India. We enrolled 5336 participants across 4220 households and began surveillance in July 2018 for viral respiratory infections with additional participants enrolled annually. Trained field workers collected data about individual-level and household-level risk factors at enrolment and quarterly assessed frailty and grip strength. Trained nurses surveilled weekly to identify acute respiratory infections (ARI) and clinically assessed individuals to diagnose acute lower respiratory infection (ALRI) as per protocol. Nasal and oropharyngeal swabs are collected from all ALRI cases and one-fifth of the other ARI cases for laboratory testing. Cost data of the episode are collected using the WHO approach for estimating the economic burden of seasonal influenza. Handheld tablets with Open Data Kit platform were used for data collection.

Findings to date The attrition of 352 participants due to migration and deaths was offset by enrolling 680 new entrants in the second year. All four sites reported negligible influenza vaccination uptake $(0.1 \%-0.4 \%)$, low health insurance coverage $(0.4 \%-22 \%)$ and high tobacco use $(19 \%-52 \%)$. Ballabgarh had the highest proportion $(54.4 \%)$ of households in the richest wealth quintile, but reported high solid fuel use (92\%). Frailty levels were highest in Kolkata (11.3\%) and lowest in Pune (6.8\%). The Chennai cohort had highest self-reported morbidity (90.1\%).

Future plans The findings of this cohort will be used to inform prioritisation of strategies for influenza and RSV control for older adults in India. We also plan to conduct epidemiological studies of SARS-CoV-2 using this platform.
Strengths and limitations of this study

- It is a multiyear, geographically spread multisite community-based cohort with weekly surveillance for respiratory viral infections; covering a wide variety of risk factors measured using standard tools; and is supported by reverse transcriptasePCR-capable labs that participate in regular quality control.

- The cohort has demonstrated low attrition in the first year since enrolment began and uses information technology-enabled solutions with standard protocols allowing real-time monitoring of data collection.

- One key limitation is the use of modified British Thoracic Society's definition for community-acquired pneumonia (excluding auscultation) as there are no clear case definitions of pneumonia meant for use in primary care setting.

- One of the unforeseen challenges for this cohort has been the onset of the SARS-CoV-2/COVID-19 pandemic with resultant lockdowns and movement restrictions.

\section{INTRODUCTION}

Community-acquired pneumonia is one of the leading contributors of mortality among older adults globally, and viral pathogens like influenza virus and respiratory syncytial virus (RSV) are common causes of pneumonia in this age group. ${ }^{12}$ These infections result in significant morbidity, hospital admissions and disability, and declines in function and quality of life. ${ }^{34}$ An estimated $54 \%-70 \%$ of all influenza-associated hospitalisations and $71 \%-85 \%$ of all influenza deaths in the USA occur among adults aged $\geq 65$ years with an 
estimated economic burden of US $\$ 8.3$ billion in the year $2013 .{ }^{56}$ Before the COVID-19 pandemic, $40 \%$ of hospitalisations with acute respiratory infection (ARI) in China occurred among those $>65$ years of age with a mean estimated cost of US $\$ 2735$ per episode in adults aged $>60$ years. ${ }^{78}$ The annual number of hospital admissions for RSV infections in older adults worldwide had been estimated at 336000 hospitalisations, with about 14000 in-hospital deaths. ${ }^{9}$

As per the 2011 census, older adults (aged $\geq 60$ years) account for $8 \%$ (104 million) of the total population of India; this proportion is projected to reach $19 \%$ by the year 2050, representing one-sixth of the total number of older adults in the world. ${ }^{10}$ An estimated $2.9 \%$ of deaths in people aged $>70$ years in India are caused by respiratory infections, and $11.6 \%$ are attributable to chronic respiratory conditions. ${ }^{10}$ Using the excess mortality approach, Narayan et al estimated influenza-associated excess respiratory deaths among persons aged $\geq 65$ years to be 34275 (95\% CI 6178 to 62 371) in India. ${ }^{11}$ A study from India reported high rates of influenza-associated hospitalisations, ranging from 6.5 to $30.1 / 10000$ persons, among older adults aged $\geq 60$ years. ${ }^{12}$ Both studies highlighted geographical variation in influenza burden in India. There is little information about RSV-related morbidity and mortality among older adults in India, as studies on respiratory viruses in India have focused on children.

India currently conducts sentinel-based surveillance for influenza through its integrated disease surveillance programme. However, hospital-based surveillance underestimates the total burden of influenza because many older adults do not seek hospital care during severe respiratory illnesses because of frailty or poor access to care. ${ }^{13} \mathrm{In}$ low/middle-income countries such as India, health facilities do not routinely test for respiratory viruses because such tests do not guide clinical management. Such health seeking and testing practices limit the utility of hospitalbased surveillance for quantifying the disease burden of respiratory viruses among older adults. Instead, multisite population-based cohorts with active surveillance systems are better able to quantify illness associated with respiratory viruses including influenza and RSV in this age group. The CARES cohort in eastern China, established to quantify the burden of influenza and its effect on frailty and functional status among older persons, exemplifies this approach. ${ }^{14}$ The burden estimates among elderly will help inform policy-makers to consider efforts like influenza vaccination to reduce the disease and economic burden among this large proportion of population. Therefore, we initiated a multicentric community-dwelling cohort of older adults or elderly (aged $\geq 60$ years) named 'Indian Network of Population-Based Surveillance Platforms for Influenza and Other Respiratory Viruses among the Elderly (INSPIRE)' to quantify the incidence, risk factors, health seeking and cost from specified respiratory viral illness (caused by influenza and RSV) among older adults in India. We present here the study protocol and description of the cohort.

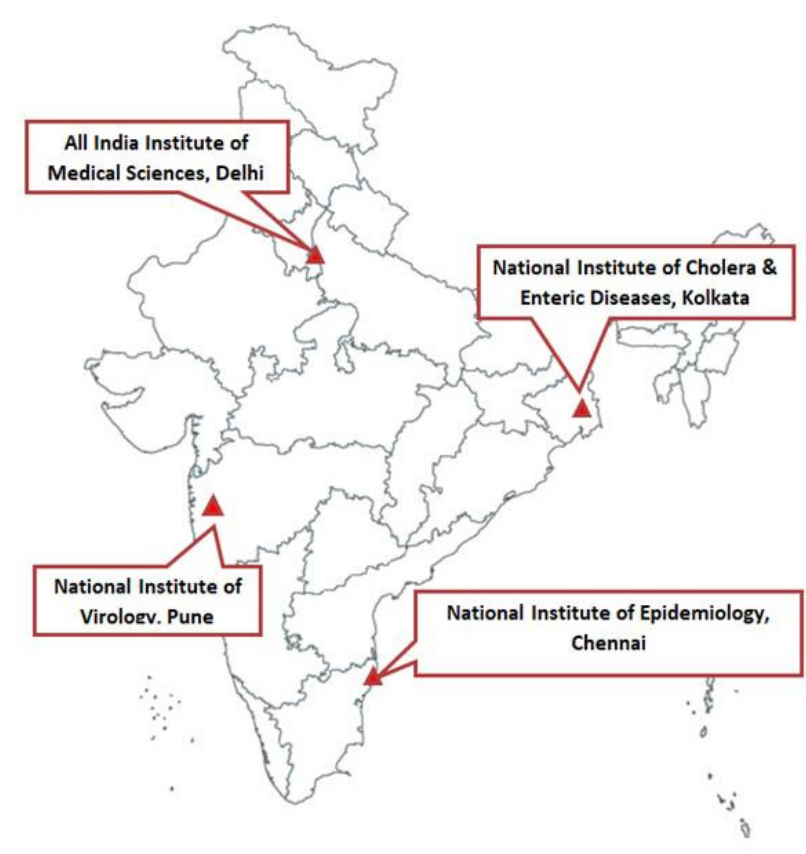

Figure 1 Map of India showing the geographical distribution of study institutions and associated sites.

\section{COHORT DESCRIPTION}

This is an ongoing dynamic cohort of community-dwelling older adults aged $\geq 60$ years in four different parts of India. Four publicly funded national institutions- the National Institute of Virology in Pune (west), National Institute of Epidemiology in Chennai (south), National Institute of Cholera and Enteric Diseases in Kolkata (east) and All India Institute of Medical Science (AIIMS) in Delhi (north) are participating. AIIMS Delhi acts as the coordination site. The INSPIRE network began enrolling participants in May 2018 and began surveillance for symptomatic respiratory infections on 2 July 2018.

\section{Study areas}

The participating institutions in Pune, Chennai, Kolkata and Delhi are shown in the map (figure 1). Each institution identified a nearby site where they were already engaged with the community. AIIMS Delhi's Ballabgarh study site is the only rural site, while cohorts in Chennai, Pune and Kolkata are in urban areas. Though influenza detection is seen throughout the year in all sites, the peak influenza activity is between June and July in Kolkata, July and September in Delhi, July and August in Pune and November and December in Chennai corresponding with rainy season in the area. A minor winter peak is seen in Delhi in January-February. ${ }^{15}$ The influenza incidence and hospitalisation was found to be higher in rural cohort near Pune than in Ballabgarh. ${ }^{16}$

\section{Sample size}

Assuming an annual cumulative incidence of influenzaassociated acute lower respiratory infection (ALRI) as 0.01 episodes per elderly individual (based on previous estimates from an earlier cohort at AIIMS, New Delhi); 
$10 \%$ annual lost to follow-up; and alpha error of 0.05 , a sample size of 4500 person years per site was calculated to estimate influenza-associated ALRI incidence with $30 \%$ relative precision. Assuming follow-up of 3 years, we required a minimum of 1500 participants at each site, with 6000 participants overall.

\section{Eligibility screening, consent, enrolment and baseline assessment of participants}

At each site, mapping and listing of all structures and the households in the study area was carried out in a structured format. At each of the listed households, the names and ages of older adults aged $\geq 60$ years who had been living in the village for more than 6 months, and with reported intention of continuing to live there, were noted by trained field workers with the help of local community volunteers. Each of the listed older adults was visited, and written informed consent was sought from them for participating in the study using a two-stage process. In the first stage, the participant was briefed about the study and screened using a four-item tool, the Dementia Assessment Rapid Test validated for Indian settings, to test cognitive impairment. ${ }^{17}$ Those assessed to be cognitively unimpaired were given detailed information about the study and its requirements, and written informed consent was obtained. The cognitively impaired were referred to nearest health facility for further evaluation. At the time of enrolment, each study participant was given a unique study identification number (participant ID). We surveyed households in the same area to recruit newly eligible individuals $\geq 60$ years old and sought their consent after screening for cognitive impairment. We collected their baseline data and began surveillance.

Our study timelines and procedures are depicted in figure 2. The key objectives, variables and tools for measurement as well as the data analysis plan are shown in table 1 .

As a dynamic cohort, additional participants are recruited annually from the listed households in the study area. During this period, individuals who were not contacted earlier or had previously declined consent, are also approached again and enrolled if they are eligible and provide consent. The sociodemographic variables studied (listed in table 1) were assessed at the baseline by trained medical social workers.

\section{ARI surveillance}

The surveillance process is described in figure 2 and the definitions used are given in box 1. At each site, trained project nurses conduct domiciliary surveillance 5 days of the week by visiting all households with enrolled participants. Each enrolled subject is visited on a fixed day of the week and asked about cough, breathlessness, or fever in the last 7 days, which synchronises with the nurse's last visit. If a participant reports any of these symptoms, the nurse performs a detailed clinical assessment including vital signs: temperature, respiratory rate, pulse rate and oxygen saturation using pulse oximeter. Based on the definition given in box 1 , cases are classified as acute upper respiratory infection (AURI) or ALRI. If the participant has ALRI or oxygen saturation of less than $90 \%$, the nurse refers the participant to the study physician for appropriate management or suggests a visit to the nearest health facility. On each surveillance day, one nurse out of five is randomly selected to collect swabs from all AURI specimens. Nurses use flocked swabs to collect midturbinate nasal and oropharyngeal specimens as per standard protocol for virology testing from all ALRI cases. The swabs are placed immediately into viral transport media on ice/icepack and are triple-sealed for transportation to the virology laboratory of the study institute on the same day. Specimens are assigned laboratory numbers using a barcode system that links to the unique study identification number. If a participant is not available at the residence at the time of the nurse's visit, but is expected to come back, another visit is made to the household on the same day. We also allow reporting of symptoms by proxy from a household adult member and note the provider of information. During the next weekly visit, if the patient was symptomatic in the previous week, follow-up questions to identify the end of the episode are asked. If symptoms persist, a repeat clinical examination is conducted to identify worsening of the illness, but repeat swabbing is not done if symptoms have continued.

\begin{tabular}{|l|l|l|l|l|l|l|l|l|l|l|l|l|l|l|l|}
\hline & & \multicolumn{7}{|c|}{ Timelines (3 monthly quarter) } \\
\hline Activity & Frequency & Start & Q1 & Q2 & Q3 & Q4 & Q5 & Q6 & Q7 & Q8 & Q9 & Q10 & Q11 & Q12 \\
\hline $\begin{array}{l}\text { Enrolment (Listing, } \\
\text { Screening, Consent) }\end{array}$ & Annual & $\bullet$ & & & & & $\bullet$ & & & & $\bullet$ & & \\
\hline $\begin{array}{l}\text { Socio-Demographic and } \\
\text { behavioral risk assessment }\end{array}$ & Once & & $\bullet$ & & & & & & & & & & & \\
\hline ARI surveillance & Weekly & & $\bullet$ & $\bullet$ & $\bullet$ & $\bullet$ & $\bullet$ & $\bullet$ & $\bullet$ & $\bullet$ & $\bullet$ & $\bullet$ & $\bullet$ & $\bullet$ \\
\hline Frailty and Grip Strength & Quarterly & & $\bullet$ & $\bullet$ & $\bullet$ & $\bullet$ & $\bullet$ & $\bullet$ & $\bullet$ & & & & & \\
\hline Cost Data Collection & Weekly & & $\bullet$ & $\bullet$ & $\bullet$ & $\bullet$ & $\bullet$ & $\bullet$ & $\bullet$ & $\bullet$ & $\bullet$ & $\bullet$ & $\bullet$ & $\bullet$ \\
\hline
\end{tabular}

Figure 2 INSPIRE study timelines and data collection procedures. INSPIRE, Indian Network of Population-Based Surveillance Platforms for Influenza and Other Respiratory Viruses among the Elderly. The figure shows the plan for data collection for burden and riskfactors for every three-monthly quarter(Q). ARI, acute respiratory infection 
Table 1 Summary of INSPIRE study objectives and methodology

\begin{tabular}{lc}
\hline Objective & Parameters measured \\
\hline Incidence of influenza-associated & ARI episodes classified \\
and respiratory syncytial virus & as acute lower respiratory \\
(RSV)-associated acute respiratory & infections (ALRI) and acute \\
infection (ARI) outpatient clinic & upper respiratory tract \\
visits and hospitalisations & infections (AURI). \\
& Care-seeking: non-medically \\
& attended, ambulatory care, \\
& hospitalisation. \\
& Influenza and RSV positivity.
\end{tabular}

Tools and methods

Weekly surveillance and clinical examination of participants with ARI; oropharyngeal/nasal swab collection in all pneumonia and $20 \%$ subsample of others; RT-PCR for influenza and RSV

Analysis plan

- AURI positivity from sample based on age group and monthwise positivity.

- Incidence of influenza/ RSV-associated ARI, acute lower respiratory infection and hospitalisations to be calculated as event/personyears at risk.

- Andersen-Gill method will be used to generate Cls for all incidence estimates.

Annual cost of influenzaassociated acute respiratory infections from the societal perspective
- Place of treatment: public/ private; primary/secondary/ tertiary.

- Direct medical costs: consultation, laboratory, procedures, medicines, consumables, room.

- Direct non-medical costs: transportation, lodging, food of participant and caregiver(s).

- Indirect costs: opportunity cost of time of participant and caregiver.

\section{Identification of risk factors for} influenza-associated and RSVassociated ALRI, hospitalisation

\section{- Household level:} socioeconomic status, solid fuel use, environmental tobacco smoke, number of children, hand washing facility, ventilation, overcrowding, wealth index.

- Individual level: age, sex, marital status, education, occupation, tobacco and alcohol use, influenza vaccination, comorbidities, anthropometry, social capital, self-rated health.
Estimation of the effect of influenza and RSV infection on frailty and cognition
- Frailty.

- Grip strength (kg).
Structured questionnaire to participant/caregiver supported by bills;

WHO/CHOICE estimates for cost of public health facilities
- Cost from all three domains (direct medical/non-medical and indirect) added to get the total cost of an episode.

- The median total cost per episode and IQR will be calculated.

- Subgroup analysis will be done to estimate cost on basis of severity of episode by type of care and by type of facility utilised (cost of influenza associated ARI in private/public health facility).

\section{Structured interview schedule to} participant adapted from various sources
Analysis for risk factors for influenza and RSV-associated ALRIwill be attempted after pooling data from the four study sites.

- Incidence rate ratio (IRR) and adjusted IRR will be estimated using generalised estimating equations with a Poisson distribution.

- Appropriate adjustments to test statistics will be made to account for repeat measures and clustering within households.

- Mean scores calculated at baseline and quarterly.

- A generalised linear model will be used with change in frailty score and grip strength in each quarter as the dependent variable and influenza/RSV illness in the interim period as the independent variable with adjustment for confounders.

INSPIRE, Indian Network of Population-Based Surveillance Platforms for Influenza and Other Respiratory Viruses among the Elderly; RT-PCR, reverse transcriptase-PCR.

During the surveillance visits, study nurses also record any hospitalisation, deaths or emigration of the participants. For each hospitalisation, date and place of admission, diagnosis and date of discharge are noted. In case of a death, the home of the deceased is visited after a gap of few weeks to conduct a verbal autopsy using a validated tool. Trained study physicians assign the cause of death. Any reported migrations are also confirmed by the medical social workers.

\section{Cost assessment}

All ALRI cases and AURI cases whose specimens are collected are asked about cost incurred by the participant for treatment of that episode. The details of the costs incurred are given in table 1 and include direct medical, direct non-medical and indirect costs. The process of data collection is described in figure 2. The cost data are collected until 1 week after the end date of the last symptom. We modelled our data collection on the WHO 


\section{Box 1 Definitions used in the cohort study}

Acute respiratory infection (ARI): New onset or worsening of cough or breathing difficulty in the last 7 days before the visit. Worsening of cough is defined as appearance of sputum, or change in colour of sputum, or increase in amount of sputum, or appearance of blood with sputum in the last 7 days as compared with the previous week

- Acute lower respiratory infection (ALRI): Adapted from the British Thoracic Society's guideline for community-acquired pneumonia diagnosis by excluding the requirement of chest auscultatory findings to increase the definition's sensitivity and feasibility in community setting. ${ }^{17}$ Symptom of acute lower respiratory tract illness (cough and at least one among breathlessness, wheeze or chest pain), tachypnoea (respiratory rate $>20$ ) and at least one systemic feature (measured temperature of $38^{\circ} \mathrm{C}$ or more or reported fever with sweating, shivers, aches and pain).

- Acute upper respiratory infections (AURI): Participants with ARI who do not meet the case definition for ALRI are considered to have AURI.

Manual for estimating the economic burden of seasonal influenza. ${ }^{18}$ Cost details are collected separately for services used at public and private health facilities. Information on costs borne out-of-pocket or billed costs for insured persons is collected for private health facilities. For public health facilities, out-of-pocket cost is supplemented by the cost of service provision as estimated by updated WHO-CHOICE. ${ }^{19}$ The appropriateness of these WHO-CHOICE estimates will be verified through review of literature and the actual cost estimation in select public health facilities. Indirect costs incurred by the affected elderly and unpaid caregivers will be estimated using a human capital approach. ${ }^{20}$

At baseline, we collected data related to socioeconomic status, overcrowding, solid fuel use, exposure to tobacco smoke, availability of hand washing facility, ventilation, comorbid conditions and influenza vaccination status. Social capital of the participants were also assessed by collecting information on social interactions, civic engagements and interpersonal trust. ${ }^{21}$ Weight, height and arm span of participants were also measured at baseline.

\section{Frailty and grip strength assessment}

Frailty assessment is carried out using the Edmonton Frailty Scale at baseline and every 3 months. ${ }^{22}$ The tool was translated into local languages and pretested. We follow the British Geriatric Society classification; participants with a score of 8-9 are considered mildly frail, whereas those with a score of $\geq 10$ (out of maximum score 17) are categorised as moderate to severely frail. ${ }^{23}$ Simultaneously, grip strength is also measured using a Jamar hand hydraulic dynamometer following the standard operating procedures. ${ }^{24}$ Each hand's grip strength is measured two times and the dominant hand is noted. Study nurses also inquire about other factors which can affect frailty and grip strength, like diagnosis of a chronic or acute condition, accidents, bereavement, history of surgery, pain or arthritis in either hand in the previous quarter.
The full study instruments are given as online supplemental materials.

\section{Patient and public involvement}

Patients or the public were not involved in the design, or conduct, or reporting, or dissemination plans of our research

\section{Laboratory procedures}

The combined mid-turbinate nasal and oropharyngeal specimens are processed, and three aliquots are prepared: one is tested and the other two aliquots are stored at $-72^{\circ} \mathrm{C}$. The specimens are tested using real-time reverse transcriptase PCR (RT-PCR) for detection, typing, subtyping and lineage of influenza viruses (A (H1N1) pdm09, A (H3N2), B (Victoria) and B (Yamagata) viruses). All sites use US Centers for Disease Control and Prevention (CDC) primers and probes available through International Reagent Resource and follow CDC protocols for influenza testing. ${ }^{25}$ Specimens are also tested for RSV using standard CDC protocols for non-influenza respiratory viruses. ${ }^{26}$ The site laboratories send the cycle threshold values and final interpretation for the specimens tested in standardised electronic form to the site data manager for linkage with surveillance data.

All sites have already successfully tested proficiency panels from the reference laboratories at AIIMS, New Delhi and CDC. Annual testing of proficiency panels will continue as part of external quality control mechanisms. Ten per cent of all positive specimens and $1 \%$ of negative specimens are tested by the reference laboratory at AIIMS, New Delhi as part of internal quality control mechanisms.

\section{Quality control}

Data collection tools were developed and standardised during all site investigators' meetings. These tools were translated into local languages, pretested and backtranslated. Standard operating procedures were developed for data collection, interviewing, clinical assessment, specimen collection and data management. The field, lab and data management staff were trained in various aspects of the project before its initiation. Periodic evaluation, retrainings and monitoring visits are conducted to ensure adherence to surveillance protocols. Local site investigators as well as central team investigators make regular visits to the field to supervise data collection. Regular teleconferences are held with the site investigators and the research team to discuss emerging field issues or changes in tools or data collection procedures. Web-based platforms are used by the central team to train new recruits as well as on-the-job training of the existing staff.

\section{Data management and analysis plan}

All data collection (except verbal autopsy) is done on handheld tablets (Android based) using the Open Data Kit platform. Step-by-step instructions for completion of the tools are included with the electronic forms. Each participant under surveillance is identified in the 
database by a unique participant ID. Barcodes are used to identify laboratory specimens, and these are used to link epidemiological and laboratory data. All data are synchronised daily to a local computer (using MySQL server) at each site. Trained computer programmers run daily and weekly checks on these data to ensure completeness, delete duplications and run logical checks for identification of outliers or unusual values. Deaths and migrations are updated weekly and the updated database is used to generate support files. These, in turn, are used in the data collection process. All changes to the data during routine cleaning are documented to create an audit trail.

Laboratory data in the form of PCR run files are imported into software developed in-house. The software facilitates laboratory scientists' inferences especially as multiple PCR runs sometimes take place, for example, for retesting and subtyping. The software output is imported into MySQL. Data files generated from merging tables in MySQL are used for interim analyses and are reviewed by the central laboratory team before being finalised. Tablets for data entry and electronic data are securely maintained (via locked storage and password protection), with access provided only to study staff who are directly involved in data management procedures. The surveillance data are merged with the database weekly after the validation checks. Encrypted data backups are created weekly and saved on an external hard drive and in cloud storage. All datasets are finalised centrally and then shared with study sites.

Based on cohort members' household assets, we calculate the wealth index using principal component analysis by pooling the households of all sites into one dataset, and calculate a score for each household. Our plan for analysis for each of objective is described in table 1. In short, we will estimate the incidence of influenza/RSV-associated events using person-years at risk as the denominator. Direct medical, direct non-medical and indirect costs will be combined to estimate total cost of illness and will be analysed across various subgroups and by sites. Risk factors for incident symptomatic influenza will be identified using adjusted incidence rate ratios using generalised estimating equations with a Poisson distribution, with appropriate adjustments for repeat measures and household-level clustering. Mean Edmonton Frail Scale and grip strength scores will be calculated at baseline and quarterly thereafter. A generalised linear model will be used to assess changes in frailty score and grip strength each quarter as the dependent variable and influenza/RSV illness in the interim period as the independent variable, with adjustment for confounders.

CDC deferred its ethical clearance to the participating institutions. We screened the participants for their cognitive ability to give consent. ${ }^{27}$ As specimens are tested in batches for optimal use of laboratory kits, results are delayed and do not guide clinical management.

\section{Findings to date}

The details of enrolment at all the four sites are provided in figure 3. Based on the initial census, the study sites identified 6560 potential participants for our target enrolment of 6000 subjects. Among these, 1026 (15.6\%) could not be contacted or did not provide consent and $98(3 \%)$ were found to have cognitive impairment. As of July 2018, $5336(81.3 \%)$ participants were consented, enrolled and surveyed at baseline and were included in the analysis. During the first year (2 July 2018-30 June 2019), 352 (6.6 $\%$ ) cohort participants were lost because of migration $(169,3.2 \%)$, death $(167,3.1 \%)$ and refusal to continue surveillance $(16,0.3 \%)$. The attrition was lowest in the rural site of Ballabgarh $(2.9 \%)$ and similar in the other three sites $(8.6 \%-9.1 \%)$. The attrition was more than compensated for by enrolment of new eligible participants $(n=680)$ in the beginning of second year, taking the total enrolment to 5664 participants.

The baseline characteristics of the cohort at each site are shown in table 2. Pune's cohort had the lowest proportion $(19.9 \%)$ of participants aged 70 years and above, and Ballabgarh the highest $(37.6 \%)$. The majority of participants in all sites were women $(57.5 \%$ overall). Overall, half $(51.6 \%)$ of the participants did not have any formal schooling, with highest proportion in Kolkata $(66.8 \%)$ and lowest in Chennai (25.1\%). Ninety per cent of the Chennai participants reported comorbidity compared with $53.9 \%$ in Delhi. Almost $50 \%$ of the participants used tobacco (smoked and smokeless) except in Chennai, where only $18.9 \%$ reported tobacco use. Mean body mass index was highest in the Chennai cohort (24.8, SD 4.7) and lowest in Ballabgarh (22.4, SD 4.7). Coverage with influenza vaccine was negligible across all sites $(0.2 \%)$. Ballabgarh (18\%) and Chennai (22\%) reported modest coverage with health insurance while Pune $(0.4 \%)$ and Kolkata (2\%) participants reported low coverage. Moderate to severe frailty (Edmonton Frail Scale score $\geq 10$ ) was noted in about one tenth $(9.3 \%)$ of the participants, with Pune reporting the lowest $(6.8 \%)$ and Kolkata the highest $(11.3 \%)$. Availability of mobile phone with a household member was reported to be highest in Ballabgarh (94.3\%) followed by Chennai (93.7\%), Kolkata (92.8\%) and lowest in Pune (86.6\%).

Overall, 92\% of households in Ballabgarh (the only rural site) reported use of solid fuels compared with $34 \%$ in Chennai and around $15 \%$ in the other two sites. In terms of economic characteristics, Kolkata households were the poorest, with only $62.5 \%$ having a dwelling that has finished material used in its walls, flooring and roof, $66.2 \%$ reporting having water sealed toilet facility within the house, which were near universal in the other sites. The highest proportion (54.3\%) of the Ballabgarh households fell into the wealthiest quintiles compared with only $0.5 \%$ of Kolkata and $0.1 \%$ Pune households.

During the first year of surveillance, 2009 nasal and oropharyngeal specimens were processed for influenza and influenza virus was detected in $4.9 \%(037 / 762$ specimens) at Ballabgarh, $5.1 \%(021 / 408$ specimens $)$ at 


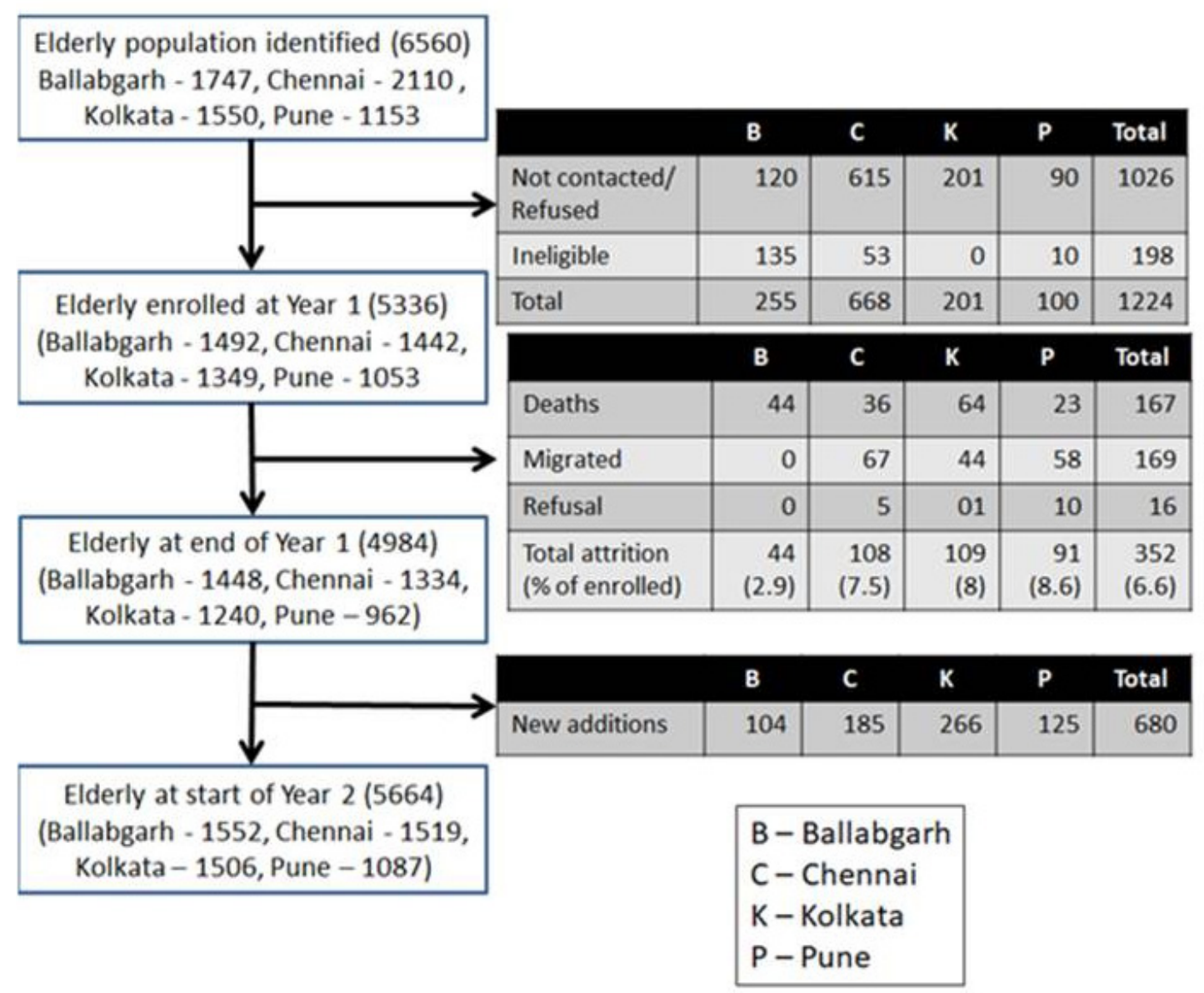

Figure 3 Flow chart of cohort enrolment and retention till first year.

Chennai, $9.5 \%$ (045/472 specimens) at Kolkata and $7.1 \%$ $(026 / 367)$ at Pune.

\section{Strengths and limitations}

Our study has considerable strengths. It is a multi-year, multi-site community-based cohort with weekly surveillance for respiratory viral infections; covers a wide variety of risk factors measured using standard tools; and is supported by RT-PCR-capable labs that participate in regular quality control. The cohort has demonstrated low attrition in the first year since enrolment began and uses information technology-enabled solutions with standard protocols allowing real-time monitoring of data collection.

One key limitation is the use of modified British Thoracic Society's (BTS) definition for communityacquired pneumonia (excluding auscultation). There are no clear case definitions of pneumonia meant for use in primary care setting. Clinical case definitions of pneumonia in primary care were originally devised as a part of the primary healthcare package for HIV/AIDS under the Integrated Management of Adolescent and Adult Illnesses (IMAI). IMAI defined pneumonia as presence of any two of the following signs: fast breathing $(\geq 20$ per min), night sweats, and chest pain in a subject with cough. ${ }^{28}$ This case definition had a sensitivity of $100 \%$ and specificity of $96.6 \%$ as compared with a physician diagnosis in Ethiopia. ${ }^{29} \mathrm{~A}$ recent meta-analysis of clinical features for diagnosis of pneumonia among adults in primary care setting identified respiratory rate $\geq 20$ per min (pooled positive likelihood ratios 3.47; 95\% CI 1.46 to 7.23$)$ and temperature $\geq 38^{\circ} \mathrm{C}(3.21 ; 95 \%$ CI 2.36 to $4.23)$ as the two best clinical predictors of pneumonia. ${ }^{30}$ These support our decision to use the modified BTS case definition for the study.

Though the four sites are geographically and socioeconomically diverse, they do not represent all older adults of India. Although we aimed to enrol 6000 participants the first year, we only enrolled $5336(89 \%)$ at baseline because of the smaller study population at Pune.

One of the unforeseen challenges for this cohort has been the onset of the SARS-CoV-2/COVID-19 pandemic with resultant lockdowns and movement restrictions. These restrictions resulted in the interruption of data and respiratory specimen collection from 25 March 2020 to 1 September 2020. During the period, syndromic surveillance was carried out by interviewing participants over phone. The surveillance has restarted from October 2020 and will continue for 1 year more.

\section{Future plans}

As demographic transition progresses, India's-as well as the world's-population of elderly will increase and health priorities, which are already transitioning from 
Table 2 Key baseline characteristics of the study participants by site

\begin{tabular}{|c|c|c|c|c|c|}
\hline Characteristics & Ballabgarh & Chennai & Kolkata & Pune & Total \\
\hline \multicolumn{6}{|l|}{ Individual characteristics } \\
\hline $\mathrm{N}$ & 1492 & 1442 & 1349 & 1053 & 5336 \\
\hline \multicolumn{6}{|l|}{ Age in years } \\
\hline $60-69$ & 931 (62.4\%) & $989(68.6 \%)$ & $1064(78.9 \%)$ & $844(80.1 \%)$ & $3828(71.7 \%)$ \\
\hline$\geq 70$ & $561(37.6 \%)$ & $453(31.4 \%)$ & $285(21.1 \%)$ & 209 (19.9\%) & $1508(28.3 \%)$ \\
\hline Male & $628(42.1 \%)$ & $646(44.8 \%)$ & $570(42.3 \%)$ & $426(40.5 \%)$ & $2270(42.5 \%)$ \\
\hline Without any formal schooling & $922(61.8 \%)$ & $362(25.1 \%)$ & $901(66.8 \%)$ & $568(53.9 \%)$ & $2753(51.6 \%)$ \\
\hline Self-reported morbidity ${ }^{\star}$ & 804 (53.9 \%) & $1299(90.1 \%)$ & $1017(75.4 \%)$ & $657(62.4 \%)$ & 3777 (70.8\%) \\
\hline Mean \pm SD BMI in $\mathrm{kg} / \mathrm{m}^{2}$ & $\begin{array}{l}22.4 \pm 4.7 \\
(n=1483)\end{array}$ & $\begin{array}{l}24.8 \pm 4.7 \\
(n=1387)\end{array}$ & $\begin{array}{l}23.5 \pm 5.2 \\
(n=1266)\end{array}$ & $\begin{array}{l}23.5 \pm 4.6 \\
(n=1033)\end{array}$ & $\begin{array}{l}23.5 \pm 4.9 \\
(n=5169)\end{array}$ \\
\hline Current tobacco use & 700 (46.9\%) & $272(18.9 \%)$ & $701(52.0 \%)$ & 547 (52.0\%) & $2220(41.6 \%)$ \\
\hline Ever receipt of influenza vaccine & $1(0.1 \%)$ & $6(0.4 \%)$ & $3(0.2 \%)$ & $3(0.3 \%)$ & $13(0.2 \%)$ \\
\hline Covered by health insurance & $268(18.0 \%)$ & $318(22.1 \%)$ & $27(2 \%)$ & $4(0.4 \%)$ & $617(11.6 \%)$ \\
\hline Moderately or severely frail $\dagger$ & $147(9.9 \%)$ & $124(8.6 \%)$ & $153(11.3 \%)$ & $72(6.8 \%)$ & $496(9.3 \%)$ \\
\hline $\begin{array}{l}\text { Availability of mobile phone with a household } \\
\text { member }\end{array}$ & $1407(94.3 \%)$ & $1351(93.7 \%)$ & $1252(92.8 \%)$ & $912(86.6 \%)$ & $4922(93.6 \%)$ \\
\hline \multicolumn{6}{|l|}{ Household characteristics } \\
\hline $\mathrm{N}$ & 1099 & 1096 & 1179 & 846 & 4220 \\
\hline Use of solid fuel in the household $\neq$ & $1011(92.0 \%)$ & $378(34.5 \%)$ & $195(16.5 \%)$ & $122(14.4 \%)$ & $1706(40.4 \%)$ \\
\hline Pucca house§ & $1036(94.3 \%)$ & $948(86.5 \%)$ & $737(62.5 \%)$ & 829 (98.0\%) & $3550(84.1 \%)$ \\
\hline Presence of water-sealed toilet facility & $1002(91.2 \%)$ & $1060(96.7 \%)$ & $781(66.2 \%)$ & $828(97.9 \%)$ & $3671(87.0 \%)$ \\
\hline Households in highest quintileq & $597(54.3 \%)$ & $190(17.3 \%)$ & $6(0.5 \%)$ & $1(0.1 \%)$ & $848(18.8 \%)$ \\
\hline
\end{tabular}

*Includes diabetes, hypertension, chronic heart, liver, kidney or respiratory disease, stroke, arthritis, malignancy, anaemia, depression, tuberculosis.

$\dagger$ †dmonton Frail Scale score $\geq 10$.

¥Solid fuels include wood, coal, shrubs, dung cakes.

§'Pucca house' is a local term referring to a dwelling that has finished material used in its walls, flooring and roof.

IThe Wealth Index was calculated for the entire cohort, divided into quintiles and then applied to individual households across cohort sites. BMI, body mass index.

a focus on maternal and child health to chronic noncommunicable disease, may focus more on the needs of older adults. The WHO's '10 Priorities for a Decade of Action on Healthy Ageing' proposes better aligning of research for healthy ageing with the needs of policymakers, clinicians and older people. ${ }^{31}$ WHO's priority 4 promotes research that addresses the current and future needs of older people.

There are not many large elderly cohorts in India, and the INSPIRE cohort with its network of public institutions with associated virology laboratories is a very useful adjunct to other national cohorts, with a focus on communicable diseases of interest in elderly. The INSPIRE cohort can be used to study other health priorities of older adults as well.

Currently, the government in India recommends influenza vaccination for healthcare workers, pregnant women and those with comorbid conditions, and not among the elderly. ${ }^{32}$ A major barrier to recommending influenza or pneumococcal vaccination among elderly is that there is no population-based vaccination platform for adults in the country. Though hospital-based vaccination is offered, its coverage is extremely limited. The recently launched publicly funded initiative of National Programme for Healthcare of the Elderly provides a possible platform for introducing influenza related public health interventions like vaccination. ${ }^{33}$ Based on the epidemiological and economic data generated from our INSPIRE cohort, we plan to evaluate the marginal value of investing in prevention and control measures for common respiratory pathogens. This would help the policy-makers in taking informed decision on adopting such measures as a part of recommendation and national programmes. Since the INSPIRE platform provides community-based surveillance for respiratory infections in elderly people in India, we are planning to expand surveillance to include SARS-CoV-2 infections. This will help assess changing illness, infection and presentation to healthcare for other respiratory infections during the pandemic. 
Author affiliations

${ }^{1}$ Centre for Community Medicine, All India Institute of Medical Sciences, New Delhi, India

${ }^{2}$ Department of Microbiology, All India Institute of Medical Sciences, New Delhi, India

${ }^{3}$ Influenza program, US Centers for Disease Control and Prevention, New Delhi, India

${ }^{4}$ National Institute of Epidemiology, Chennai, India

${ }^{5}$ National Institute of Cholera and Enteric Diseases, Kolkata, India

${ }^{6}$ National Institute of Virology, Pune, India

${ }^{7}$ Influenza division, US Centers for Disease Control and Prevention, Atlanta, Georgia, USA

Acknowledgements We acknowledge the support of all the investigators and coinvestigators, our research staff, study participants and other members of the community who helped us in conducting this study.

Collaborators This study was collaboration between AllMS (New Delhi), NIE (Chennai), NIV (Pune), NICED (Kolkata) and CDC, Atlanta. We are open to collaboration with other researchers to use data generated in this study for global estimates as well as to use the platform to conduct further research on influenza and other respiratory viruses.

Contributors AK, LD, KL, EA-B, SS, RA and RK conceptualised the study. RA and $\mathrm{AOP}$ prepared the data collection tools and trained the field staff in the study protocol. LD, AsC, AvC and SD prepared laboratory testing protocol and provided external quality control to site laboratory. AK, RA, SS, AOP, RK and GGP provided technical oversight to study sites. AK, RK, RA and GGP supervised field data collection at Ballabgarh site. LD and AsC supervised laboratory testing while AvC and SD conducted testing at Ballabgarh site. PR and CPGK were the investigators from Chennai site. PR supervised field data collection and CPGK supervised laboratory testing. SDB and VP were the investigators from Pune site. SDB supervised field data collection and VP supervised laboratory testing. SK, AC and BM were investigators from Kolkata site. SK and BM supervised field data collection while AC supervised laboratory testing in Kolkata site. Data from all sites was managed and analyzed by RA, AOP and GGP. All authors interpreted the results, contributed to writing the manuscript, and approved the final version for submission. AK is the guarantor of the study.

Map disclaimer The inclusion of any map (including the depiction of any boundaries therein), or of any geographic or locational reference, does not imply the expression of any opinion whatsoever on the part of BMJ concerning the legal status of any country, territory, jurisdiction or area or of its authorities. Any such expression remains solely that of the relevant source and is not endorsed by BMJ. Maps are provided without any warranty of any kind, either express or implied.

Competing interests None declared.

Patient consent for publication Consent obtained directly from patient(s)

Ethics approval The study was cleared by the institutional ethical committees of all the participating institutions (AllMS -IEC-283/02.06.17, NIE-IHEC/2017-03, NIV-IEC/2018/D5, NICED-A-1/2017-IEC). CDC deferred its ethical clearance to the participating institutions (Protocol No:7145).

Provenance and peer review Not commissioned; externally peer reviewed.

Data availability statement Data are available on reasonable request.

Supplemental material This content has been supplied by the author(s). It has not been vetted by BMJ Publishing Group Limited (BMJ) and may not have been peer-reviewed. Any opinions or recommendations discussed are solely those of the author(s) and are not endorsed by BMJ. BMJ disclaims all liability and responsibility arising from any reliance placed on the content. Where the content includes any translated material, BMJ does not warrant the accuracy and reliability of the translations (including but not limited to local regulations, clinical guidelines, terminology, drug names and drug dosages), and is not responsible for any error and/or omissions arising from translation and adaptation or otherwise.

Open access This is an open access article distributed in accordance with the Creative Commons Attribution Non Commercial (CC BY-NC 4.0) license, which permits others to distribute, remix, adapt, build upon this work non-commercially, and license their derivative works on different terms, provided the original work is properly cited, appropriate credit is given, any changes made indicated, and the use is non-commercial. See: http://creativecommons.org/licenses/by-nc/4.0/.

\section{ORCID iDs}

Prabu Rajkumar http://orcid.org/0000-0001-9839-800X
Giridara Gopal Parameswaran http://orcid.org/0000-0002-2238-6843

\section{REFERENCES}

1 Shi T, Denouel A, Tietjen AK, et al. Global disease burden estimates of respiratory syncytial Virus-Associated acute respiratory infection in older adults in 2015: a systematic review and meta-analysis. $J$ Infect Dis 2020;222:S577-83.

2 Morimoto K, Suzuki M, Ishifuji T, et al. The burden and etiology of community-onset pneumonia in the aging Japanese population: a multicenter prospective study. PLoS One 2015;10:e0122247.

3 McElhaney JE. The unmet need in the elderly: designing new influenza vaccines for older adults. Vaccine 2005;23 Suppl 1:S10-25.

4 Ottenbacher KJ, Graham JE, Al Snih S, Snih SA, et al. Mexican Americans and frailty: findings from the Hispanic established populations epidemiologic studies of the elderly. Am J Public Health 2009;99:673-9.

5 Reed C, Chaves SS, Daily Kirley P, et al. Estimating influenza disease burden from population-based surveillance data in the United States. PLoS One 2015;10:e0118369.

6 McLaughlin JM, McGinnis JJ, Tan L, et al. Estimated human and economic burden of four major adult vaccine-preventable diseases in the United States, 2013. J Prim Prev 2015;36:259-73.

7 Zheng J, Huo X, Huai Y, et al. Epidemiology, seasonality and treatment of hospitalized adults and adolescents with influenza in Jingzhou, China, 2010-2012. PLoS One 2016;11:e0150713.

8 Yang J, Jit M, Leung KS, et al. The economic burden of influenzaassociated outpatient visits and hospitalizations in China: a retrospective survey. Infect Dis Poverty 2015;4:44.

9 Shi T, McLean K, Campbell H, et al. Aetiological role of common respiratory viruses in acute lower respiratory infections in children under five years: a systematic review and meta-analysis. J Glob Health 2015;5:010408.

10 Registrar General of India, Centre for Global Health Research. Causes of death statistics in India 2010-2013. New Delhi. [updated December 2015], 2015. Available: http://www.cghr.org/2015/12/ causes-of-death-in-india-2010-2013/ [Accessed 30 Sep 2021]

11 Narayan VV, luliano AD, Roguski K, et al. Burden of influenzaassociated respiratory and circulatory mortality in India, 2010-2013. J Glob Health 2020;10:010402.

12 Shi T, McAllister DA, O'Brien KL, et al. Global, regional, and national disease burden estimates of acute lower respiratory infections due to respiratory syncytial virus in young children in 2015: a systematic review and modelling study. Lancet 2017;390:946-58.

13 Ortiz JR, Neuzil KM, Cooke CR, et al. Influenza pneumonia surveillance among hospitalized adults may underestimate the burden of severe influenza disease. PLoS One 2014;9:e113903.

14 Cowling BJ, Xu C, Tang F, et al. Cohort profile: the China ageing REespiratory infections study (cares), a prospective cohort study in older adults in eastern China. BMJ Open 2017;7:e017503.

15 Chadha MS, Potdar VA, Saha S, et al. Dynamics of influenza seasonality at sub-regional levels in India and implications for vaccination timing. PLoS One 2015;10:e0124122.

16 Hirve S, Krishnan A, Dawood FS, et al. Incidence of influenzaassociated hospitalization in rural communities in Western and northern India, 2010-2012: a multi-site population-based study. J Infect 2015;70:160-70.

17 Swati BSV, Manjari T, Ashima N. Dementia assessment by rapid test (dart): an Indian screening tool for dementia. Jounal of Alzheimer's Disease \& Parkinsonism 2015:5:2161-460.

18 Initiative for Vaccine Research (IVR) of the Department of Immunization VaB. WHO manual for estimating the economic burden of seasonal influenza: WHO 2016;63.

19 Stenberg K, Lauer JA, Gkountouras G, et al. Econometric estimation of WHO-CHOICE country-specific costs for inpatient and outpatient health service delivery. Cost Eff Resour Alloc 2018;16:11.

20 Jo C. Cost-Of-Illness studies: concepts, scopes, and methods. Clin Mol Hepatol 2014;20:327-37.

21 Yang YC, Boen C, Mullan Harris K, Harris KM. Social relationships and hypertension in late life: evidence from a nationally representative longitudinal study of older adults. J Aging Health 2015;27:403-31.

22 Rolfson DB, Majumdar SR, Tsuyuki RT, et al. Validity and reliability of the Edmonton frail scale. Age Ageing 2006;35:526-9.

23 British Geriatric Society. Edmonton frailty scale, 2018. Available: https://www.bgs.org.uk/sites/default/files/content/attachment/201807-05/efs.pdf

24 Reeve TE, Ur R, Craven TE, et al. Grip strength measurement for frailty assessment in patients with vascular disease and associations 
with comorbidity, cardiac risk, and sarcopenia. J Vasc Surg 2018;67:1512-20.

25 Shu B, Wu K-H, Emery S, et al. Design and performance of the CDC real-time reverse transcriptase PCR swine flu panel for detection of 2009 A (H1N1) pandemic influenza virus. J Clin Microbiol 2011;49:2614-9.

26 Fry AM, Chittaganpitch M, Baggett HC, et al. The burden of hospitalized lower respiratory tract infection due to respiratory syncytial virus in rural Thailand. PLoS One 2010;5:e15098-e98.

27 Gray WK, Paddick SM, Collingwood C, et al. Community validation of the idea study cognitive screen in rural Tanzania. Int $J$ Geriatr Psychiatry 2016;31:1199-207.

28 World Health Organization. Integrated management of adolescent and adult illness: guidelines for first-level facility health workers at health centre and district outpatient clinic. WHO/CDS/IMAl/2004.1 ed. Geneva: WHO, 2009.
29 Woldie M, Enquselassie F. Assessment of the validity of the Guideline for integrated management of adult and adolescent illnesses on HIV patients in Addis Ababa, Ethiopia. Ethiop Med J 2009;47:195-203.

30 Htun TP, Sun Y, Chua HL, et al. Clinical features for diagnosis of pneumonia among adults in primary care setting: a systematic and meta-review. Sci Rep 2019;9:7600.

31 World Health Organization. 10 priorities towards a decade of healthy ageing. Geneva: WHO, 2017.

32 National Centre for Disease Control. Seasonal influenza: guidelines for vaccination with influenza vaccine: NCDC, 2021. Available: https://ncdc.gov.in/showfile.php?lid=405

33 Directorate General of Health Services. National programme for health care of the elderly -operational guidelines Ministry of health and family welfare India 2011;42. 